\title{
1. Experimental and Theoretical Study of Selectivity in Mechanochemical Cocrystallization of Nicotinamide with Anthranilic 3 and Salicylic Acid
}

${ }_{4}$ Published as part of a Crystal Growth and Design virtual special issue Honoring Prof. William Jones and His ${ }_{5}$ Contributions to Organic Solid-State Chemistry

${ }_{6}$ Stipe Lukin, ${ }^{\dagger}$ Ivor Lončarić, $^{\dagger}$ Martina Tireli, ${ }^{\dagger}$ Tomislav Stolar, ${ }^{\dagger}{ }^{\circ}$ Maria V. Blanco, ${ }^{\ddagger}$ Predrag Lazić, ${ }^{\dagger}$

7 Krunoslav Užarević, ${ }^{\dagger}$ and Ivan Halasz ${ }^{*}{ }^{\dagger}$ (1)

$8{ }^{\dagger}$ Ruder Bošković Institute, Bijenička c. 54, 10000 Zagreb, Croatia

$9{ }^{\ddagger}$ ESRF - the European Synchrotron, 71 Avenue des Martyrs, 38000 Grenoble, France

10

Supporting Information

\begin{abstract}
Selectivity in mechanochemical cocrystal formation between nicotinamide and anthranilic acid or salicylic acid was studied using tandem in situ reaction monitoring by powder X-ray diffraction (PXRD) and Raman spectroscopy. Selectivity was probed by offering a competing cocrystal coformer to a previously prepared cocrystal or under competitive reaction conditions where all cocrystal coformers, in different stoichiometric ratios, were introduced together in the starting reaction mixture. Reaction paths were dependent
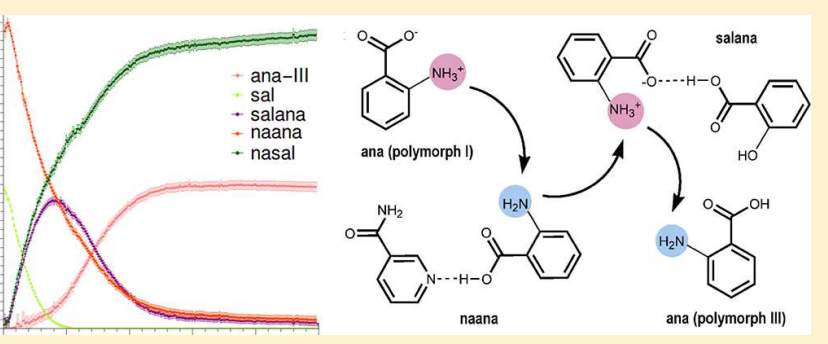
on the starting mixture composition, and we find that the

formation of intermediates and the final product can be predicted from solid-state ab initio calculations of relative energies of possible reaction mixtures. In some cases, quantitative assessment revealed different reaction profiles derived from PXRD and Raman monitoring, directly indicating, for the first time, different mechanochemical reactivity on the molecular and the bulk crystalline level of the reaction mixture.
\end{abstract}

\section{INTRODUCTION}

26 Mechanochemical reactions are becoming increasingly attrac27 tive since they offer a means to perform selective chemical 28 synthesis with excellent atom- and energy-economy and with 29 little or, in some cases, no waste generation. ${ }^{1-3}$ Being 30 previously limited largely to processing of minerals, metals, 31 alloys, and inorganic materials, ${ }^{4}$ chemical transformations 32 achievable by using mechanochemistry now encompass almost 33 every aspect of chemical synthesis, including synthesis of 34 organic, $^{5-8}$ organometallic, ${ }^{9-11}$ metal-organic com35 pounds $^{12-14}$ as well as multicomponent solids such as 36 cocrystals ${ }^{15,16}$ and the preparation of nanoparticles, ${ }^{17}$ molecular 37 nanostructures, ${ }^{18}$ pharmaceuticals, ${ }^{19}$ advanced peptide syn38 thesis, ${ }^{20,21}$ supramolecular recognition, ${ }^{22}$ and recently, enzy39 matic catalysis. ${ }^{23,24}$ The widespread use of mechanochemistry 40 has been since recently accompanied by methodology to probe 41 the course and mechanisms of milling reactions by real-time in 42 situ monitoring techniques based on X-ray diffraction, ${ }^{25,26}$ 43 Raman spectroscopy, ${ }^{9,27}$ or their simultaneous use in 44 tandem. ${ }^{28,29}$

45 These techniques have revealed surprisingly fast mechano46 chemical reactions and metastable and short-lived intermedi47 ates, indicating that mechanochemical reactions may bear a 48 strong resemblance to solution chemistry, despite them being conducted in the solid state. Moreover, we hypothesize that 49 many principles derived for solution reactions may also be valid 50 for mechanochemical reactions. In principle, we believe that a 51 surface of potential energy could be constructed where each 52 stable solid phase would lie in its minimum and where energy 53 barriers could be identified for their potential transforma- 54 tions. ${ }^{30-32}$ So far, such an energy landscape is evidenced by the 55 observation of the Ostwald's rule of stages, ${ }^{29,33}$ the equilibra- 56 tion of solids, ${ }^{34-36}$ and a stronger than expected influence of 57 temperature on mechanochemical reactions. ${ }^{37}$ Also, while the 58 catalytic effect of liquid additives is well-known, ${ }^{38,39}$ direct 59 observation of lowering of an energy barrier and hence reaction 60 acceleration by different liquid additives in a liquid-assisted 61 grinding (LAG) reaction was recently observed. ${ }^{40}$ In creating 62 parallels with solution reactions, one also needs to consider 63 solvation of solid particles, ${ }^{41}$ which seems to have enabled 64 control over selective preparation of three polymorphs of 65 nicotinamide/benzoic acid cocrystals. ${ }^{29}$

Specific energy relations between solids in an energy 67 landscape would need to result in selectivity in mechanochem- 68

Received: October 30, 2017

Revised: January 5, 2018 
69 ical reactions. Indeed, in a pioneering study, Etter has shown 70 specific pairing of nucleobases in a grinding reaction in the 71 presence of a competing nucleobase. ${ }^{42}$ Caira and co-workers 72 have also shown selective cocrystal preparation starting from a 73 mixture of cocrystal coformers, ${ }^{43}$ but they have taken their 74 study a step further in showing that an already formed cocrystal 75 will react if a more selective coformer is offered. In a more 76 recent example, we have shown strong selectivity in supra77 molecular anion binding with the ability of a host molecule to 78 discriminate even among six dicarboxylic acids. ${ }^{22}$ Abourahma 79 and co-workers have studied the robustness of a particular 80 theophylline cocrystal while grinding with other potential 81 coformers and offered a qualitative interpretation of coformer 82 replacements based on hydrogen bonding preferences. ${ }^{44}$ 83 Emmerling and co-workers have described a selectivity study 84 between several active pharmaceutical ingredients and 85 anthranilic acid as a cocrystal coformer and suggested that 86 energetic and kinetic factors may need to be taken into account 87 as well. ${ }^{45}$

Herein, we describe selectivity in mechanochemical cocrystal 89 formation between nicotinamide (na) and two benzenecarbox90 ylic acids differing in the functionality on the ortho position: 91 anthranilic acid (ana) and salicylic acid (sal) (Figure 1). Via

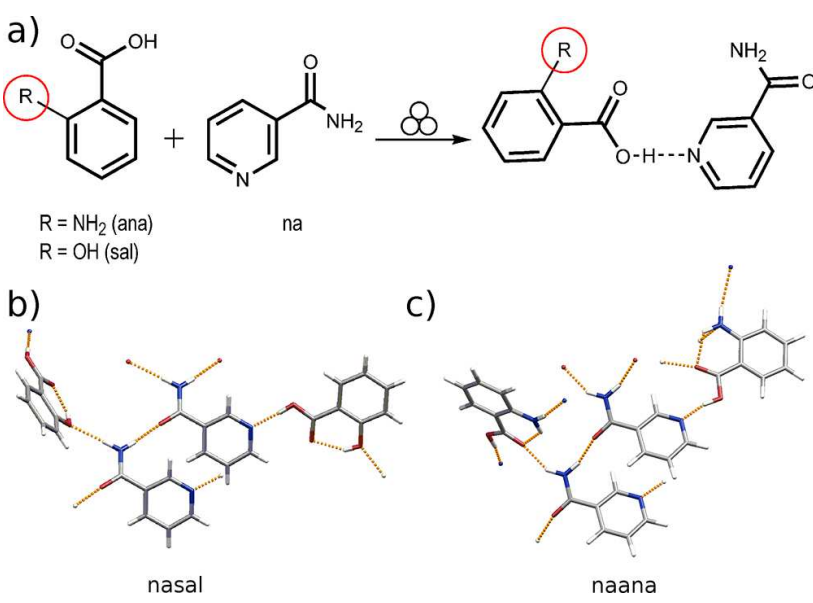

Figure 1. (a) Mechanochemical preparation of cocrystals of na with sal and ana. Hydrogen bonding in the (b) crystal structure of the nasal cocrystal $^{46}$ and (c) crystal structure of the naana cocrystal.

92 quantitative assessment by tandem in situ powder X-ray 93 diffraction and Raman monitoring, ${ }^{29}$ we have observed varied 94 and intricate reaction paths in competitive cocrystal formation 95 and cocrystal replacement reactions, involving formation of 96 metastable intermediates and multistep solid-state proton 97 transfer. Accompanied by theoretical calculations of stabilities 98 of participating solid phases, we find that the observed 99 selectivity can be readily predicted from relative stabilities of 100 cocrystals and coformers, while the reaction mechanisms may 101 nevertheless involve various, less stable intermediate phases. In 102 the course of this study we have solved, from powder diffraction 103 data, the crystal structure of the nicotinamide/anthranilic acid 104 (naana) cocrystal (Figure 1c) and revised the crystal structure 105 of the salicylic acid/anthranilic acid (salana) cocrystal.

\section{RESULTS AND DISCUSSION}

107 Cocrystal Synthesis. Anthranilic acid is known to form 108 three polymorphs. ${ }^{47}$ The one we obtained commercially and used in these experiments is designated as polymorph I (ana-I) 109 and is zwitterionic. More precisely, of the two molecules in the 110 asymmetric unit of polymorph I, one is a "normal" non- 111 zwitterionic molecule while the other is zwitterionic. Before 112 studying selectivity in multicomponent reaction mixtures, we 113 have attempted to prepare naana and nasal cocrystals 114 separately. In the 1:1 na:ana reaction mixture, the naana 115 forms readily and directly from reactants with no detectable 116 intermediates. Formation of naana begins immediately upon 117 milling, and the reaction was fully complete within $20 \mathrm{~min} 118$ milling. Crystal structure of the naana cocrystal was thus far 119 unknown, so we have solved it from powder diffraction data 120 (Figures 1c and S6). The crystal structure resembles that of the 121 known nasal cocrystal ${ }^{46}$ (Figure $1 \mathrm{~b}$ ), but it is stabilized by a 122 larger number of hydrogen bonds since the amino group of ana 123 acts both as an acceptor and a donor of hydrogen bonds. In the 124 naana cocrystal, ana is not zwitterionic. The 1:1 cocrystal 125 formation between sal and na also proceeds directly from 126 reactants, and the reaction is complete within 20 min milling. 127 Final product is the known nasal cocrystal. ${ }^{46}$ Even though both 128 reactions are complete within ca. 20 min milling, in situ 129 monitoring revealed naana formation to be somewhat faster 130 than nasal formation, suggesting a lower energy barrier for 131 naana formation (Figure 2). For both cocrystal synthesis $132 \mathrm{f} 2$ reactions, quantitative assessments based on either Raman and 133 PXRD monitoring have revealed similar reaction profiles, 134 indicating that the reaction mixture is crystalline and contains 135 little or no amorphous component.

136

Cocrystal Selectivity and Replacement Reactions. 137 Following cocrystal synthesis, we have attempted to predict 138 the selectivity in formation of each cocrystal by using ab initio 139 calculations in the solid state to estimate the relative energies of 140 cocrystals and cocrystal coformers. Ab initio calculations are 141 likely to provide sufficiently precise energies, which can be used 142 to assess possibilities of cocrystal formation ${ }^{48}$ as well as 143 polymorphic transitions. ${ }^{49}$ In particular, density functional 144 theory (DFT) with newly developed functionals that include 145 van der Waals interactions achieve good accuracy in modeling 146 molecular crystals. ${ }^{50}$ Of several van der Waals implementations 147 in DFT, here we choose the nonemipirical vdW-DF-cx 148 functional $^{51,52}$ that proved its accuracy in similar systems. ${ }^{49,53,54} 149$ Since the evaluation of (temperature dependent) Gibbs free 150 energies from DFT comes with a very high computational cost, 151 here we only report the relative enthalphies. It should be noted 152 that this could be, in addition to the exchange-correlation 153 functional, a source of imprecision in reported predictions. 154 While comparing solids with the same composition is 155 straightforward, cocrystals with different compositions can 156 also be compared, provided that the overall composition is kept 157 fixed by considering also the energy of the competing coformer 158 (Scheme 1). Such considerations should predict which $159 \mathrm{~s} 1$ cocrystal will be stable if offered a competing coformer and 160 which cocrystals will react to form a more stable cocrystal. 161

Our calculations predict all cocrystals to be more stable than 162 pure components (Scheme 1). The naana cocrystal (see later) 163 is only $0.5 \mathrm{~kJ} \mathrm{~mol}^{-1}$ more stable than the mixture of na and ana- 164 I. The salana cocrystal is by $4.0 \mathrm{~kJ} \mathrm{~mol}^{-1}$ more stable than pure 165 sal and ana-I. With $13.8 \mathrm{~kJ} \mathrm{~mol}^{-1}$ difference, the nasal cocrystal 166 is most stable of all three cocrystals relative to pure 167 components. The mixture nasal and ana-I, in the 1:1 molar 168 ratio, is the most stable combination. ${ }^{47}$ Therefore, sal should be 169 able to replace ana from the naana cocrystal, while ana should 170 not replace sal from the nasal cocrystal. 

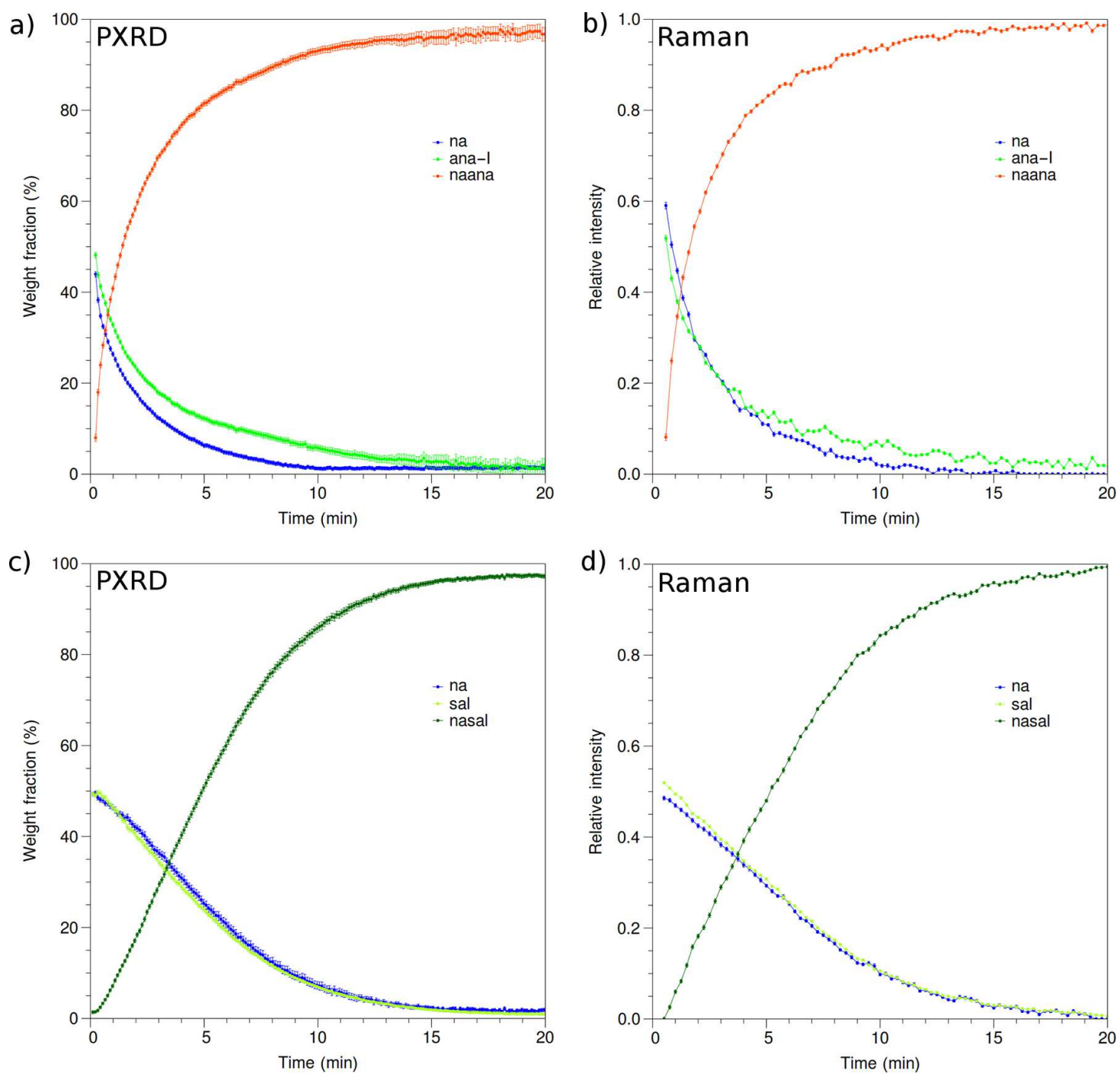

Figure 2. PXRD and Raman monitoring quantitative reaction profiles for $(a, b)$ naana and (c, d) nasal formation.

Scheme 1. Theoretical Prediction of the Order of Stability of Cocrystals and Competing Coformers in the System of na, sal, and ana ${ }^{a}$

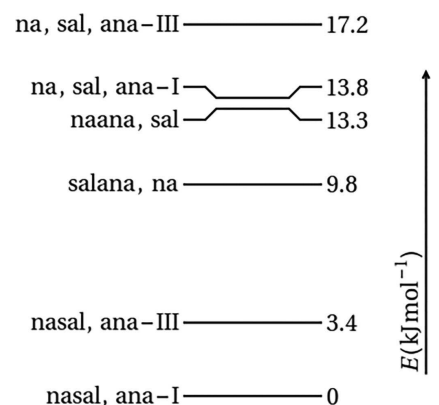

${ }^{a_{T}}$ Two polymorphs of ana are included, ana-I and ana polymorph III (ana-III).

172 For replacement experiments, a competitive cocrystal 173 coformer was added to the mechanochemically prepared 174 naana or nasal cocrystals. Thus, we have added an equimolar 175 amount of sal to the reaction vessel containing the naana 176 cocrystal and, in the second experiment, an equimolar amount of ana to the mechanochemically prepared nasal cocrystal. As 177 theory predicted, we found that ana is not able to replace sal 178 from the nasal cocrystal, but that ana is slowly becoming 179 amorphous during milling, evidenced by a gradual decrease in 180 the scale factor of ana derived from Rietveld refinement 181 (Figures S1 and S2). On the other hand, sal readily replaced 182 ana from the naana cocrystal in a reaction mechanism that 183 involved intermediate formation of salana (Figures 3 and S3). $184 \mathrm{f} 3$ The replaced ana from the naana cocrystal crystallized 185 concomitantly as a pure phase, but in a different polymorphic 186 form than the starting ana. The in situ formed ana belongs to 187 the monoclinic polymorph III of ana (ana-III), which no longer 188 has zwitterionic molecules. ${ }^{47}$ Formation of ana-III is surprising, 189 since it was previously found to transform to ana-I upon 190 grinding $^{47}$ and should be less stable than ana-I (Scheme 1). 191

Formation of ana-III provides further evidence that unusual 192 and metastable solid species may be formed in a milled reaction 193 environment. While a full investigation as to why the less stable 194 polymorph ana-III was formed here is outside the scope of this 195 work, we may offer possible explanations to its unexpected 196 formation. Nucleation and growth of ana-III during milling may 197 be in accordance with the Ostwald's rule of stages, but we have 198 not observed its transformation to the stable ana-I in the period 199 


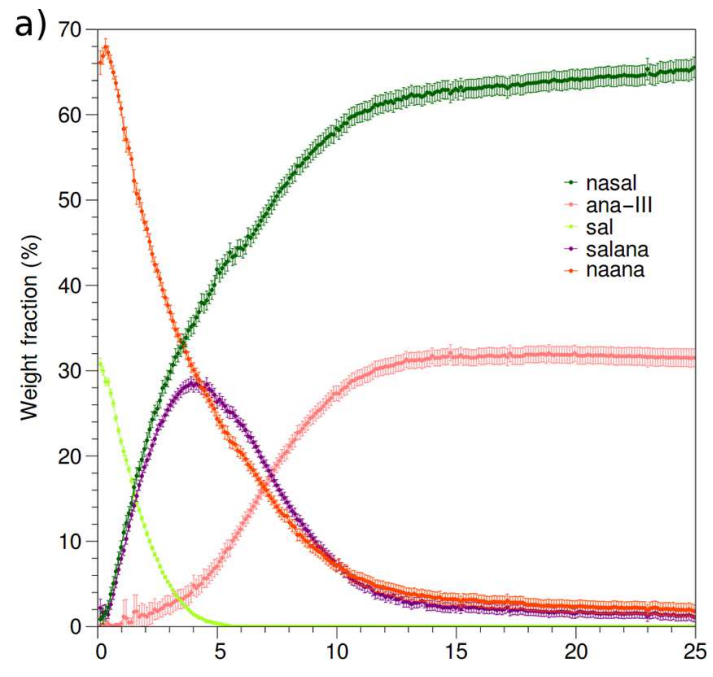

b)

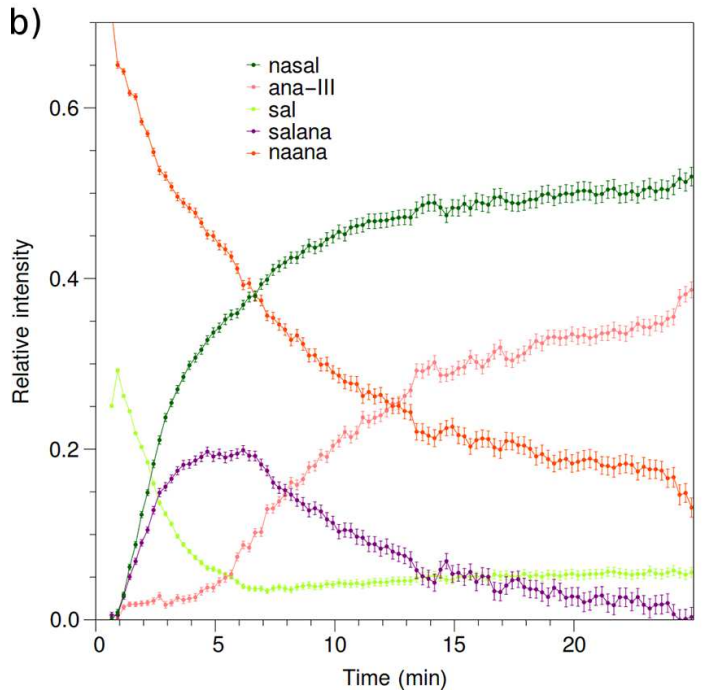

Figure 3. Reaction profiles for the mechanochemical reaction of the naana cocrystal with sal, derived from time-resolved tandem in situ monitoring by (a) PXRD and (b) Raman spectroscopy. Error bars correspond to standard deviations as obtained from the corresponding refinement procedures.

200 of 25 min milling. Formation of ana-III could be stochastic, as 201 was e.g., the case with the formation of the katsenite metal202 organic framework, ${ }^{33}$ but this is impossible to judge since the 203 experiment was repeated only once. Finally, a likely explanation 204 for the occurrence of ana-III involves stabilization of its 205 crystallite particles via surface interaction with the species 206 present in the reaction mixture, as was demonstrated by 207 Belenguer et al. ${ }^{41}$ and by us ${ }^{29}$ when using liquid additives.

208 The crystal structure of the salana cocrystal, observed here as 209 an intermediate, was recently solved as a cocrystal of sal and ana 210 molecules. $^{45}$ However, we have revised here the crystal 211 structure of salana with the amino group of ana protonated 212 in order to form three $\mathrm{N}-\mathrm{H} \cdots \mathrm{O}$ hydrogen bonds. The 213 protonated amino group leaves two options for the position 214 of one hydrogen atom: either on the carboxylic group of sal or 215 on the carboxylic group of ana. In the case of sal deprotonation, 216 a salt would be formed, while in the case the carboxylic group 217 of ana would be deprotonated, a zwitterionic cocrystal would 218 form where sal would remain nonzwitterionic and ana would become zwitterionic. Since determination of hydrogen atoms 219 from powder diffraction data may be unreliable, we have aided 220 ourselves with $\mathrm{ab}$ initio calculations to rank in energy these two 221 crystal structure candidates and found the second, zwitterionic 222 cocrystal option, to be slightly more stable. Final Rietveld 223 refinement was performed against high-resolution powder X- 224 ray diffraction data, collected at the powder diffraction beamline 225 11-BM at the Advanced Photon Source, to reveal a chemically 226 reasonable network of hydrogen bonds in the zwitterionic 227 salana cocrystal (Figures 4 and S7). a)

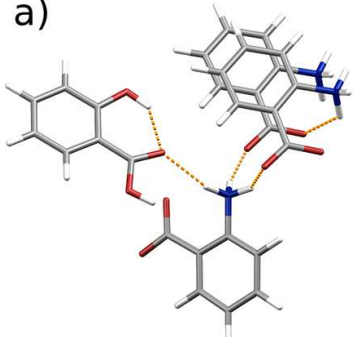

b)

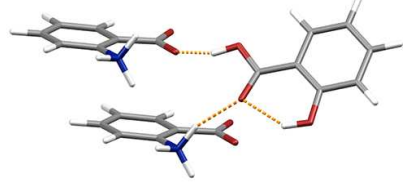

Figure 4. Hydrogen bonding in the crystal structure of the zwitterionic salana cocrystal. Hydrogen bonds are denoted with orange dashed lines. (a) Three hydrogen bonds surrounding the protonated amino group of ana and (b) the hydrogen bond between the carboxylic group of sal and the carboxylate group of ana.

With crystal structures of all participating phases now known, 229 Rietveld refinement enabled extraction of reaction profiles 230 which reveal immediate and concomitant formation of nasal 231 and salana cocrystal at approximately the same rates in the 232 beginning of the reaction, while sal is present in the reaction 233 mixture (Figure 3). Later, formation of salana gradually slows 234 down and stops just at the time pure sal is fully consumed when 235 it becomes the source of salicylic acid needed for further 236 formation of the nasal cocrystal. The crystallization of pure ana 237 then also accelerates, but, as mentioned above, it crystallizes as 238 polymorph III, rather than polymorph I. In situ Raman 239 monitoring revealed essentially the same reaction course but 240 suggests a significantly longer presence of naana and also salana, 241 which could possibly be amorphous. Worth mentioning, in this 242 reaction sequence, the amino group of ana underwent several 243 proton transfers: from the starting zwitterionic ana-I, to the 244 nonzwitterionic form in the naana cocrystal, followed again by 245 protonation in the salana cocrystal and final crystallization of a 246 nonzwitterionic ana-III (Scheme 2).

Competitive Experiments. Next to replacement reactions, 248 we have performed direct competitive experiments in mixtures 249 of na, ana-I, and sal (Figure 5). Milling equimolar amounts of $250 \mathrm{fs}$ na, sal, and ana-I resulted in the final formation of the nasal 251 cocrystal. The reaction course, however, included intermediate 252 formation of the naana cocrystal and formation of a small 253 amount of the salana cocrystal which remained in the final 254 reaction mixture. Rietveld analysis of the reaction course 255 exhibits an initial decline in the weight fraction of ana-I 256 consistent with the formation of a small amount of the naana 257 intermediaate. As naana starts to diminish after 2 min milling, 258 the weight fraction of ana-I increases, as the released ana 259 crystallizes into the starting polymorph I, before it is again 260 consumed for the formation of salana. The rate of na 261 consumption is slightly faster than the rate of consumption of 262 sal, consistent with parallel formation of nasal and naana. 263 Raman monitoring revealed the same reaction mechanism with 264 
Scheme 2. Protonation-Deprotonation Sequence of ana in the $(\mathrm{na}+$ ana $)+$ sal Reaction Sequence ${ }^{a}$

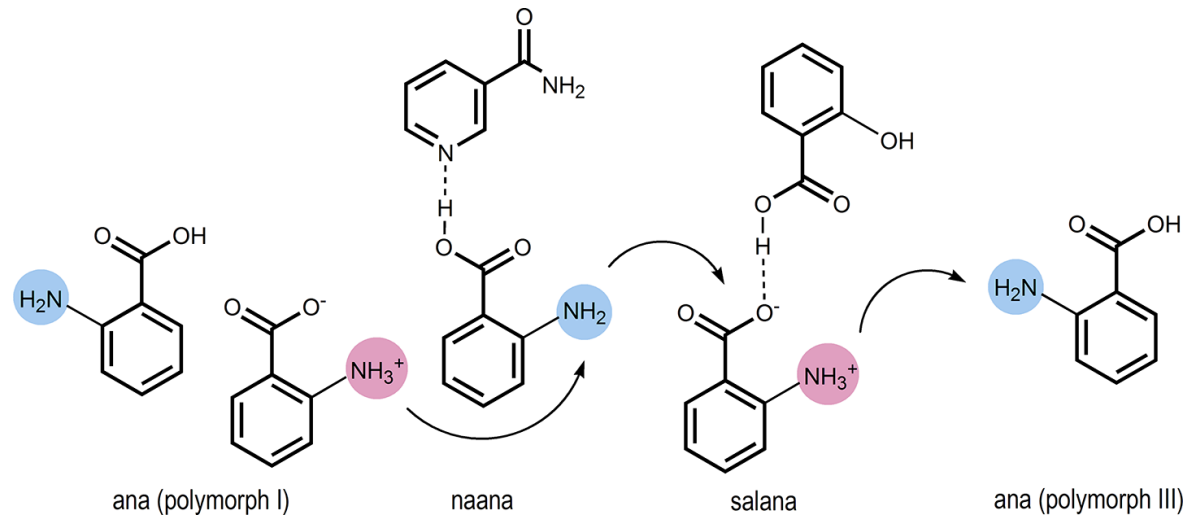

${ }^{a}$ Polymorph designation is given according to Etter. ${ }^{47}$

a)

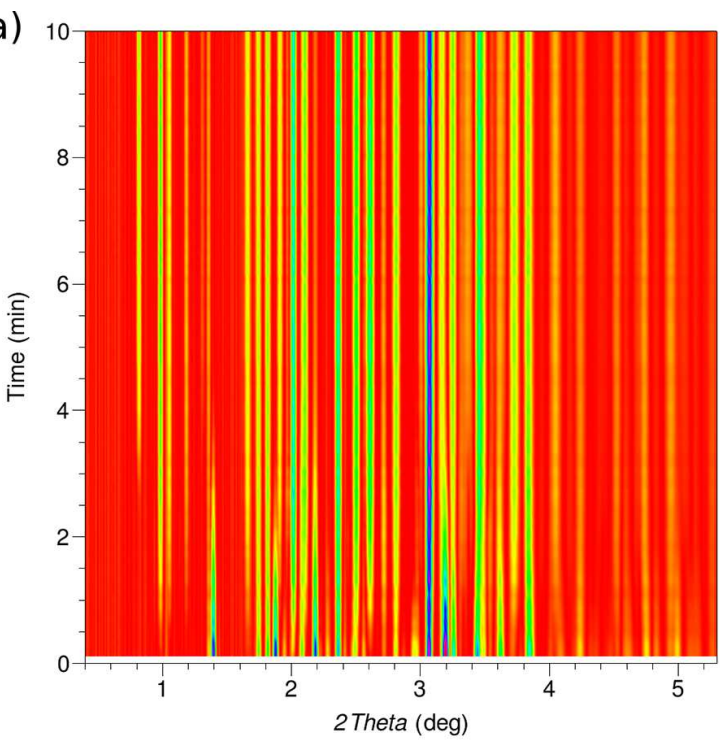

c)

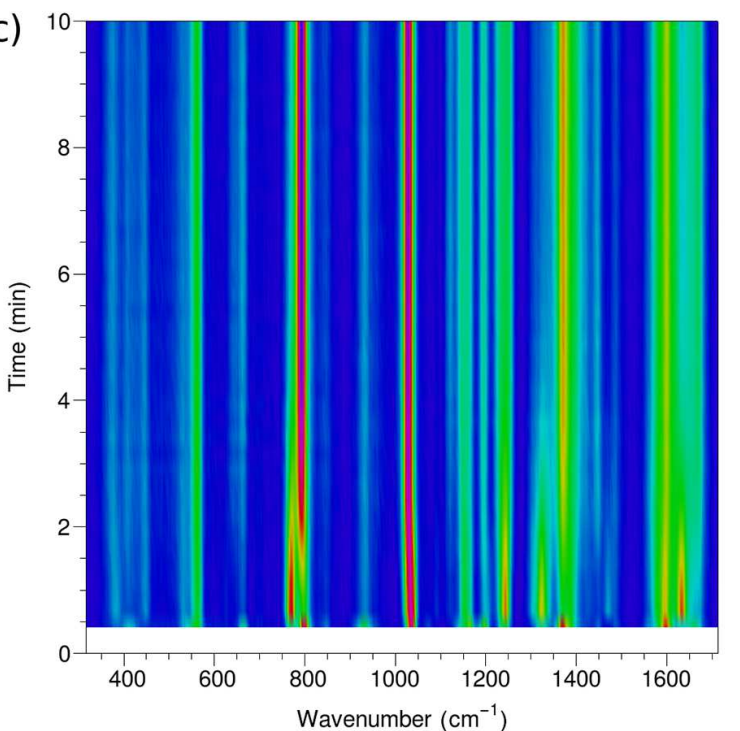

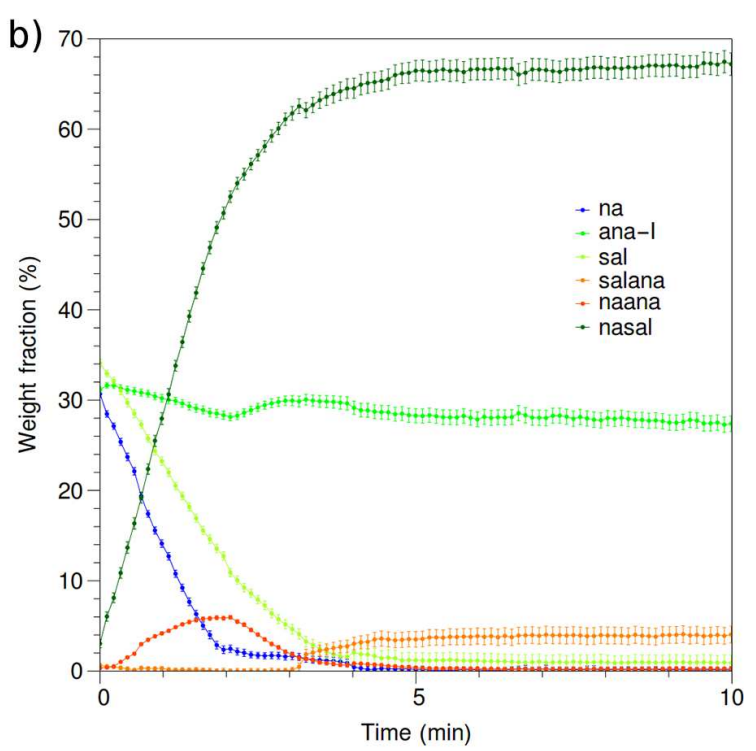

d)

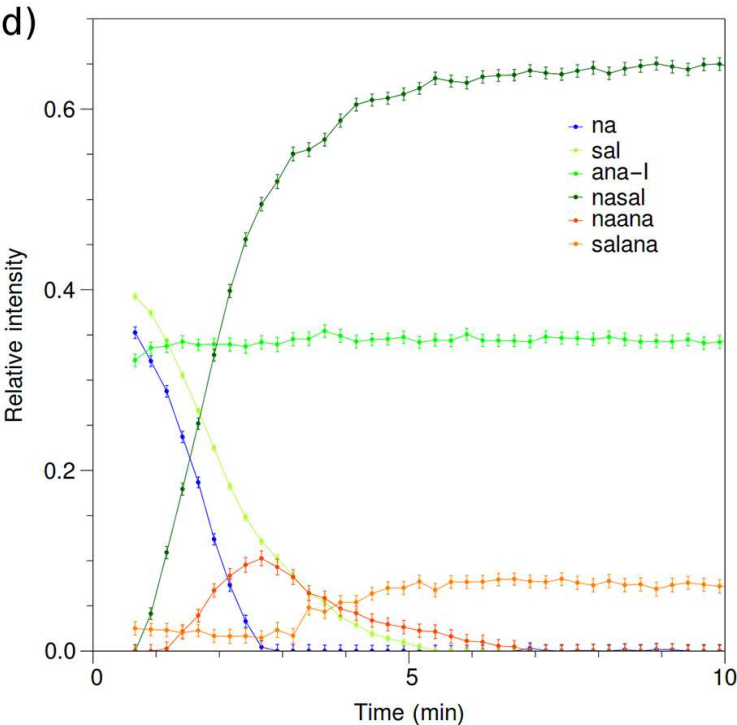

Figure 5. Competitive mechanochemical reaction of na, sal, and ana in the molar ratio 1:1:1. Tandem in situ monitoring via (a, b) PXRD and (c, d) Raman spectroscopy. (a) Two-dimensional time-resolved PXRD and (b) the corresponding reaction profile. (c) Two-dimensional time-resolved Raman spectra and (d) the corresponding reaction profile. Error bars correspond to standard deviations as obtained from the corresponding refinement procedures. 

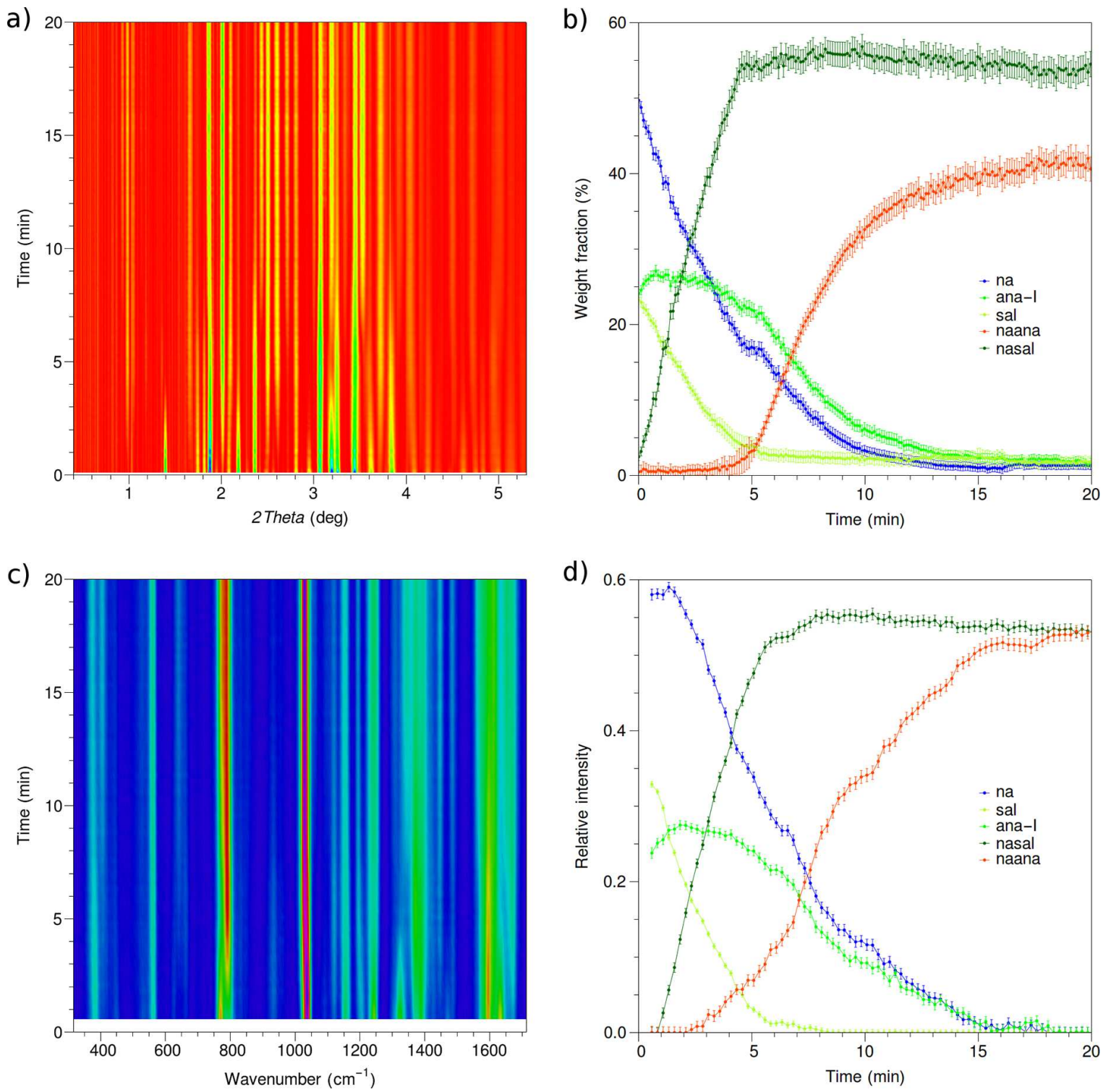

Figure 6. Competitive mechanochemical reaction of (a) na, sal, and ana in the molar ratio 1:1/2:1/2 monitored by tandem in situ (a, b) PXRD and (c, d) Raman spectroscopy. (a) Two-dimensional time-resolved PXRD and (b) the corresponding reaction profile. (c) Two-dimensional timeresolved Raman spectra and (d) the corresponding reaction profile. The reaction profiles in (b) and (d) exhibit a slight increase in the content of ana-I which is likely due to the reaction being non-homogeneous immediately after initiation of milling and thus would not correspond to an actual increase in the content of ana-I in the reaction mixture. Error bars correspond to standard deviations as obtained from the corresponding refinement procedures.

265 naana formation and a small amount of salana in the final 266 mixture. Consumption of na and sal and the formation of nasal 267 followed the same rates and trends in both PXRD and Raman 268 monitoring indicating that nasal is highly crystalline. Formation 269 of salana starts just after 3 min milling according to both Raman 270 and PXRD. naana is slightly more persistent according to 271 Raman than according to PXRD indicating the presence of 272 amorphous or nanocrystalline naana undetectable by PXRD.

273 A limited study of LAG reactions was here performed where 274 we have used methanol or propanol as liquid additives in the 275 na:sal:ana $=1: 1: 1$ reaction mixtures (Figures S4 and S5). 276 Alcohols accelerated the reactions and nasal formation was 277 finished within 5 min milling. With methanol, we observed 278 intermediate formation of naana and the formation of a small 279 amount of salana, which remained upon further milling. Using propanol as the liquid additive resulted in a similar reaction 280 mechanism, but without the formation of the intermediate 281 naana. These results strongly indicate the sensitivity of 282 mechanochemical reactions to additives and the possibility to 283 alter relative energies of solid species and barriers between 284 them and thus support the concept of the energy landscape in a 285 mechanochemical reaction environment. 286

Milling na, sal, and ana but now in the ratio of 1:1/2:1/2 287 resulted in surprisingly different reactivity as compared to the 288 1:1:1 mixture. The reaction mixture in this experiment 289 contained sufficient na to fully form both nasal and naana 290 cocrystals. Comparing PXRD and Raman monitoring (Figure $291 \mathrm{f} 6$ 6), there are several significant similarities and differences: (i) $292 \mathrm{fo}$ Formation profiles of nasal are similar in PXRD and Raman 293 monitoring, while the formation profiles of naana are 294 
295 significantly different. (ii) According to PXRD monitoring, the 296 formation of naana did not commence until crystalline sal was 297 fully consumed and nasal formation was complete. On the 298 other hand, Raman monitoring revealed naana formation soon 299 after nasal started to form. (iii) Formation curve of naana 300 derived from Raman monitoring is erratic, while it is smooth 301 according to PXRD. (iv) On the basis of the initial 302 stoichiometric ratio of reactants and the similarity of molecular 303 mases of nasal and naana cocrystals, final weight fractions of 304 nasal and naana should be similar and around 50\%. However, 305 PXRD revealed weight fraction of nasal to be around $55 \%$ and 306 weight fraction of naana around $40 \%$ (the remaining 5\% belong 307 to minor amounts of reactants). On the other hand, Raman 308 monitoring revealed, as expected, equal amounts of nasal and 309 naana at the end of milling.

310 There are several possibilities to the observed discrepancy 311 between in situ PXRD and Raman monitoring. It may be due to 312 partial amorphization of naana which would result in apparent 313 lowering of its weight fraction in Rietveld analysis, but it does 314 not explain why naana formation is not observable by PXRD 315 until all sal is consumed. Another possibility involves formation 316 of a solid solution of naana in the nasal cocrystal which would 317 increase weight fraction of nasal at the expense of naana, but 318 this explanation assumes that naana in a solid solution in nasal 319 would have the same Raman spectrum as pure ana, which may 320 be an unlikely assumption. It is also likely that naana crystal 321 growth is hindered as long as sal is present in the reaction 322 mixture, consistent with the replacement reaction of naana with 323 sal. Since Raman monitoring indicates presence of naana, it can 324 only be amorphous or nanocrystalline and thus not detectable 325 by X-ray diffraction. This is supported by a small decrease in 326 ana weight fraction before the onset of naana crystallization. 327 Only when sal is fully consumed, growth of microcrystalline 328 naana can proceed when it can be detected by PXRD. This last 329 possibility, however, does not explain the discrepancy in final 330 weight fractions of nasal and naana.

\section{$331 \square$ CONCLUSION}

332 We have described mechanochemical transformations and 333 selectivity in a system of nicotinamide cocrystals with two 334 similar aromatic carboxylic acids: salicylic acid and anthranilic 335 acid. Cocrystals show a distinct order of selectivity and a less 336 preferred one is readily converted to a preferred cocrystal when 337 the competing coformer is offered. Among various approaches 338 to explaining the order of selectivity, we find theoretical energy 339 determination to be advantageous. With the advance in 340 computational techniques and the availability of high-perform341 ance computers, we believe it is no longer necessary to resort to 342 qualitative approaches, such as Etter's rules, ${ }^{5,56}$ which in this 343 case, would suggest opposite reactivity. Theoretical calculations 344 will likely provide an order of stability with a high predictive 345 power, but we should nevertheless remain aware that in milling 346 the real sample need not conform to perfect crystals, ${ }^{57,58}$ which 347 are taken into theoretical predictions, but have defects, a 348 distribution of particle sizes, and could easily contain an 349 amorphous component.

350 This work has provided a clear example of different reaction 351 profiles derived form PXRD and Raman monitoring putting an 352 emphasis on the complementarity of the two techniques for in 353 situ and real-time monitoring of mechanochemical reactions. 354 While PXRD will detect bulk crystalline species, Raman 355 monitoring, which is more sensitive to the molecular structure 356 and the immediate molecular surroundings, will nevertheless detect a reaction occurring in an amorphous component of the 357 reaction mixture. We therefore expect Raman monitoring to 358 become an indispensable tool in understanding the dynamics of 359 the mechanochemical reaction environment.

\section{EXPERIMENTAL SECTION}

Mechanochemical reactions were carried out as described previously ${ }^{29} 362$ using a Retsch MM301 (Germany) mixer mill operating at $30 \mathrm{~Hz} .363$ Translucent and amorphous reaction vessels made from polymethyl- 364 metacrylate (PMMA) had the internal volume of $14 \mathrm{~mL}$ and were 365 purchased from InSolido Technologies (Croatia). Two halves of the 366 vessel snapped upon closure to form a leak-proof seal. Liquids in 367 liquid-assisted grinding reactions were added using a Gilson automated 368 micropipet. Tandem in situ monitoring experiments were conducted at 369 the ESRF beamline ID31 as described previously. ${ }^{29}$ Briefly, the 370 experimental hutch was air-conditioned to $20^{\circ} \mathrm{C}$, the X-ray beam and 371 the Raman laser focus were positioned to approximately coincide on 372 the same portion of the reaction mixture, X-ray radiation wavelength 373 of $0.195 \AA$ was selected using a multilayer monochromator, diffraction 374 data were recorded on a Dectris Pilatus CdTe $2 \mathrm{M}$ detector positioned 375 $1067 \mathrm{~mm}$ from the sample, radial integration of the raw diffraction 376 images was performed using PyFAI, ${ }^{59}$ and exposure time for each 377 pattern was $5.0 \mathrm{~s}$. Time resolution between consecutive diffraction 378 patterns was ca. $6.5 \mathrm{~s}$, and time resolution of Raman spectra was 379 typically $10 \mathrm{~s}$. As milling media, two $7 \mathrm{~mm}$ stainless steel balls were 380 used, each weighing $1.4 \mathrm{~g}$.

Crystal structure of naana was solved from powder X-ray diffraction 382 data collected on a laboratory Panalytical instrument in the Bragg- 383 Brentano geometry. Structure was solved by simulated annealing in 384 direct space using known molecular fragments of na and ana, which 385 were treated as rigid bodies. Crystal structure solution was recognized 386 when a meaningful hydrogen bond was assembled between molecular 387 fragments of na and ana and with no close contacts between 388 nonbonded atoms. The structure model was finally refined treating 389 molecules of na and ana as rigid bodies. The crystal structure of salana 390 was previously reported as a cocrystal of nonzwitterionic molecules of 391 sal and ana ${ }^{45}$ and is here revised as a zwitterionic cocrystal where the 392 ana molecule in the cocrystal is zwitterionic. The new structure model 393 of salana cocrystal was refined against high-resolution synchrotron 394 powder diffraction data, collected at the 11-BM beamline of the 395 Advanced Photon Source, using restraints on bond distances and 396 angles as well as planarity restraints. All calculations were performed 397 using the program Topas. Crystal structures of salana and naana are 398 deposited with the Cambridge Crystallographic Data Center (CCDC) 399 under deposition numbers 1581782 and 1581783 and can be retrieved 400 from CCDC upon request.

401

Quantitative Rietveld refinement ${ }^{60}$ was performed on a series of in 402 situ collected powder diffraction patterns in an automated fashion 403 using batch files and the command-line version of Topas, either always 404 starting from the same input file or in a sequential manner. ${ }^{26,29} 405$ Rietveld refinements included refinement of the parameters for the 406 shifted Chebyshev polynomial used to describe the background 407 parameters, parameters contributing the peak position and shape 408 (contribution to the Lorenzian and Gaussian full widths at half- 409 maximum, zero shift, unit cell parameters). No instrument 410 contribution to peak shape was assumed. Upon convergence of 411 Rietveld refinement for each pattern, the obtained relative crystalline 412 phase weight fractions were output to a separate file and used for 413 further plotting. All plots were created using the program Mathematica 414 with the help of the SciDraw package. ${ }^{61}$ For two-dimensional time 415 -resolved plots, the background of each diffraction pattern was 416 subtracted prior to plotting using the Sonneveld-Visser ${ }^{62}$ algorithm 417 implemented in Mathematica. Spectral range of $316-1713 \mathrm{~cm}^{-1}$ of 418 Raman spectra were taken for analysis. Reaction vessel subtraction and 419 baseline subtraction in Raman spectra was performed as previously 420 described. $^{29}$ Raman spectra of pure phases of na, sal, ana-I, ana-III, 421 nasal, naana, and salana were collected in the same experimental 422 conditions. ana-III was prepared as described elsewhere. ${ }^{47}$ Raman 423 
424 reaction profiles were derived using nonnegative classic least-squares 425 approach implemented in MATLAB. ${ }^{63}$

426 DFT calculations were performed using a plane-wave basis set code 427 Quantum Espresso ${ }^{64}$ with GBRV pseudopotentials ${ }^{65}$ and vdW-DF-cx 428 exchange-correlation functional. ${ }^{51,52}$ The plane-wave basis set cutoff 429 was set to $820 \mathrm{eV}$, and the first Brillouin zone was sampled by a $3 \times 3$ $430 \times 3$ Monkhorst-Pack k-point mesh. In each calculation, crystal lattice 431 and atom positions were relaxed until the change in the total energy 432 was $<0.5 \mathrm{meV}$, all the forces were smaller than $0.01 \mathrm{eV} / \AA$, and 433 pressure was $<0.5 \mathrm{kbar}$.

\section{ASSOCIATED CONTENT}

\section{(5) Supporting Information}

436 The Supporting Information is available free of charge on the 437 ACS Publications website at DOI: 10.1021/acs.cgd.7b01512.

$438 \quad$ PXRD and Raman spectra; Rietveld fit data; crystallo-

439 graphic ad refinement data (PDF)

\section{Accession Codes}

441 CCDC 1581782-1581783 contain the supplementary crystal442 lographic data for this paper. These data can be obtained free of 443 charge via www.ccdc.cam.ac.uk/data_request/cif, or by email444 ing data_request@ccdc.cam.ac.uk, or by contacting The 445 Cambridge Crystallographic Data Centre, 12 Union Road, 446 Cambridge CB2 1EZ, UK; fax: +44 1223336033.

\section{AUTHOR INFORMATION}

\section{${ }_{448}$ Corresponding Author}

449 *E-mail: ivan.halasz@irb.hr.

450 ORCID $\odot$

451 Tomislav Stolar: 0000-0002-9824-4462

452 Krunoslav Užarević: 0000-0002-7513-6485

453 Ivan Halasz: 0000-0002-5248-4217

\section{Notes}

455 The authors declare no competing financial interest.

\section{ACKNOWLEDGMENTS}

457 Financial support from the Croatian Science Foundation 458 (Grant No. UIP-2014-09-4744) is gratefully acknowledged. $459 \mathrm{I} . \mathrm{H}$. is grateful to the Adris foundation for supporting this work. 460 S.L. is supported by the Croatian Science Foundation. I.L. and 461 P.L. are supported by the Unity Through Knowledge Fund, 462 Contract No. 22/15 and the H2020 CSA Twinning Project No. 463692194, RBI-T-WINNING. We are grateful to the team at the 464 fine-mechanics workshop of the Ruder Boškovic Institute for 465 their continuous support and the staff of the 11-BM beamline 466 for powder data collection of the salana cocrystal. Use of the 467 Advanced Photon Source at Argonne National Laboratory was 468 supported by the U.S. Department of Energy, Office of Science, 469 Office of Basic Energy Sciences, under Contract No. DE-AC02470 06CH11357.

\section{REFERENCES}

472 (1) James, S. L.; et al. Mechanochemistry: opportunities for new and 473 cleaner synthesis. Chem. Soc. Rev. 2012, 41, 413-447.

474 (2) Kaupp, G.; Schmeyers, J.; Boy, J. Waste-free solid-state syntheses 475 with quantitative yield. Chemosphere 2001, 43, 55-61.

476 (3) Do, J.-L.; Friščić, T. Chemistry 2.0: Developing a New, Solvent477 Free System of Chemical Synthesis Based on Mechanochemistry. 478 Synlett 2017, 28, 2066-2092.

479 (4) Takacs, L. The historical development of mechanochemistry. 480 Chem. Soc. Rev. 2013, 42, 7649-7659.
(5) Stolle, A.; Szuppa, T.; Leonhardt, S. E. S.; Ondruschka, B. Ball 481 milling in organic synthesis: solutions and challenges. Chem. Soc. Rev. 482 2011, 40, 2317-2329.

(6) Kaupp, G. Organic solid-state reactions with $100 \%$ yield. Top. 484 Curr. Chem. 2005, 254, 95-183. 485

(7) Wang, G.-W. Mechanochemical organic synthesis. Chem. Soc. Rev. 486 2013, 42, 7668-7700.

(8) Hernández, J. G.; Bolm, C. Altering Product Selectivity by 488 Mechanochemistry. J. Org. Chem. 2017, 82, 4007-4019. 489

(9) Juribašíc, M.; Užarević, K.; Gracin, D.; Curić, M. Mechanochem- 490 ical C-H bond activation: rapid and regioselective double cyclo- 491 palladation monitored by in situ Raman spectroscopy. Chem. Commun. 492 2014, 50, 10287-10290.

(10) Hernández, J. G. C-H Bond Functionalization by Mechano- 494 chemistry. Chem. - Eur. J. 2017, 23, 17157-17165. 495

(11) Hermann, G. N.; Becker, P.; Bolm, C. Mechanochemical 496 Rhodium(III)-Catalyzed C-H Bond Functionalization of Acetanilides 497 under Solventless Conditions in a Ball Mill. Angew. Chem., Int. Ed. 498 2015, 54, 7414-7417.

(12) Pichon, A.; Lazuen-Garay, A.; James, S. L. Solvent-free synthesis 500 of a microporous metal-organic framework. CrystEngComm 2006, 8, 501 211-214.

(13) Stolar, T.; Batzdorf, L.; Lukin, S.; Žilić, D.; Motillo, C.; Friščíć, 503 T.; Emmerling, F.; Halasz, I.; Užarević, K. In Situ Monitoring of the 504 Mechanosynthesis of the Archetypal Metal-Organic Framework 505 HKUST-1: Effect of Liquid Additives on the Milling Reactivity. 506 Inorg. Chem. 2017, 56, 6599-6608.

507

(14) Užarević, K.; Wang, T. C.; Moon, S.-Y.; Fidelli, A. M.; Hupp, J. 508 T.; Farha, O. K.; Friščíc, T. Mechanochemical and solvent-free 509 assembly of zirconium-based metal-organic frameworks. Chem. 510 Commun. 2016, 52, 2133-2136.

(15) Braga, D.; Maini, L.; Grepioni, F. Mechanochemical preparation 512 of co-crystals. Chem. Soc. Rev. 2013, 42, 7638-7648.

(16) Hasa, D.; Schneider Rauber, G.; Voinovich, D.; Jones, W. 514 Cocrystal Formation through Mechanochemistry: from Neat and 515 Liquid-Assisted Grinding to Polymer-Assisted Grinding. Angew. Chem., 516 Int. Ed. 2015, 54, 7371-7375.

(17) Xu, C.; De, S.; Balu, A. M.; Ojeda, M.; Luque, R. 518 Mechanochemical synthesis of advanced nanomaterials for catalytic 519 applications. Chem. Commun. 2015, 51, 6698-6713.

(18) Içli, B.; Christinat, N.; Tönnemann, J.; Schuttler, C.; Scopelliti, 521 R; Severin, K. Synthesis of Molecular Nanostructures by Multi- 522 component Condensation Reactions in a Ball Mill. J. Am. Chem. Soc. 523 2009, 131, 3154-3155.

(19) Tan, D.; Loots, L.; Friščíc, T. Towards medicinal mecha- 525 nochemistry: evolution of milling from pharmaceutical solid form 526 screening to the synthesis of active pharmaceutical ingredients (APIs). 527 Chem. Commun. 2016, 52, 7760-7781.

(20) Bonnamour, J.; Metro, T.-X.; Martinez, J.; Lamaty, F. 529 Environmentally benign peptide synthesis using liquid-assisted ball- 530 milling: application to the synthesis of Leu-enkephalin. Green Chem. 531 2013, 15, 1116-1120.

532

(21) Konnert, L.; Gauliard, A.; Lamaty, F.; Martinez, J.; Colacino, E. 533 Solventless Synthesis of N-Protected Amino Acids in a Ball Mill. ACS 534 Sustainable Chem. Eng. 2013, 1, 1186-1191.

535

(22) Užarević, K.; Halasz, I.; Đilović, I.; Bregović, N.; Rubčić, M.; 536 Matković-Čalogović, D.; Tomišíc, V. Dynamic Molecular Recognition 537 in Solid State for Separating Mixtures of Isomeric Dicarboxylic Acids. 538 Angew. Chem., Int. Ed. 2013, 52, 5504-5508. 539

(23) Hernandez, J. G.; Ardila-Fierro, K. J.; Crawford, D.; James, S. L.; 540 Bolm, C. Mechanoenzymatic peptide and amide bond formation. 541 Green Chem. 2017, 19, 2620-2625.

542

(24) Pérez-Venegas, M.; Reyes-Rangel, G.; Neri, A.; Escalante, J.; 543 Juaristi, E. Mechanochemical enzymatic resolution of N-benzylated- $\beta^{3}$ - 544 amino esters. Beilstein J. Org. Chem. 2017, 13, 1728-1734. 545

(25) Friščić, T.; Halasz, I.; Beldon, P. A.; Belenguer, A. M.; Adams, 546 F.; Kimber, S. A. J.; Honkimäki, V.; Dinnebier, R. E. Nat. Chem. 2013, 547 $5,66-73$. 
549 (26) Halasz, I.; Friščić, T.; Kimber, S. A. J.; Užarević, K.; Puškarić, A.; 550 Mottillo, C.; Julien, P.; Štrukil, V.; Honkimäki, V.; Dinnebier, R. E. 551 Quantitative in situ and real-time monitoring of mechanochemical 552 reactions. Faraday Discuss. 2014, 170, 203-221.

553 (27) Gracin, D.; Štrukil, V.; Friščić, T.; Halasz, I.; Užarević, K. 554 Laboratory Real-Time and In Situ Monitoring of Mechanochemical 555 Milling Reactions by Raman Spectroscopy. Angew. Chem., Int. Ed. 556 2014, 53, 6193-6197.

557 (28) Batzdorf, L.; Fischer, F.; Wilke, M.; Wenzel, K.-J. r.; Emmerling, 558 F. Direct In Situ Investigation of Milling Reactions Using Combined 559 X-ray Diffraction and Raman Spectroscopy. Angew. Chem., Int. Ed. 560 2015, 54, 1799-1802.

561 (29) Lukin, S.; Stolar, T.; Tireli, M.; Blanco, M. V.; Babić, D.; Friščić, 562 T.; Užarević, K.; Halasz, I. Tandem In Situ Monitoring for 563 Quantitative Assessment of Mechanochemical Reactions Involving 564 Structurally Unknown Phases. Chem. - Eur. J. 2017, 23, 13941-13949. 565 (30) Jansen, M.; Doll, K.; Schoen, J. C. Addressing chemical diversity 566 by employing the energy landscape concept. Acta Crystallogr., Sect. A: 567 Found. Crystallogr. 2010, 66, 518-534.

568 (31) Jansen, M. The energy landscape concept and its implications 569 for synthesis planning. Pure Appl. Chem. 2014, 86, 883-898.

570 (32) Andersen, J. M.; Mack, J. Decoupling the Arrhenius equation via 571 mechanochemistry. Chem. Sci. 2017, 8, 5447-5453.

572 (33) Katsenis, A. D.; Puskarić, A.; Štrukil, V.; Mottillo, C.; Julien, P. 573 A.; Užarević, M. H.; Pham, K.; Do, T. O.; Kimber, S. A. J.; Lazić, P.; 574 Magdysyuk, O.; Dinnebier, R. E.; Halasz, I.; Friščić, T. Nat. Commun. 575 2015, 6, 6662 .

576 (34) Belenguer, A. M.; Friščić, T.; Day, G. M.; Sanders, J. K. M. 577 Solid-state dynamic combinatorial chemistry: reversibility and 578 thermodynamic product selection in covalent mechanosynthesis. 579 Chem. Sci. 2011, 2, 696-700.

580 (35) Bygrave, P. J.; Case, D. H.; Day, G. M. Is the equilibrium 581 composition of mechanochemical reactions predictable using 582 computational chemistry? Faraday Discuss. 2014, 170, 41-57.

583 (36) Belenguer, A. M.; Lampronti, G. I.; Wales, D. J.; Sanders, J. K. $584 \mathrm{M}$. Direct Observation of Intermediates in a Thermodynamically 585 Controlled Solid-State Dynamic Covalent Reaction. J. Am. Chem. Soc. 586 2014, 136, 16156-16166.

587 (37) Užarević, K.; Štrukil, V.; Mottillo, C.; Julien, P. A.; Puškarić, A.; 588 Friščić, T.; Halasz, I. Exploring the Effect of Temperature on a 589 Mechanochemical Reaction by in Situ Synchrotron Powder X-ray 590 Diffraction. Cryst. Growth Des. 2016, 16, 2342-2347.

591 (38) Shan, N.; Toda, F.; Jones, W. Mechanochemistry and co-crystal 592 formation: effect of solvent on reaction kinetics. Chem. Commun. 2002, $5932372-2373$.

594 (39) Halasz, I.; Puškarić, A.; Kimber, S. A. J.; Beldon, P. J.; Belenguer, 595 A. M.; Adams, F.; Honkimäki, V.; Dinnebier, R. E.; Patel, B.; Jones, 596 W.; Štrukil, V.; Friščić, T. Angew. Chem., Int. Ed. 2013, 52, 1153859711541.

598 (40) Tireli, M.; Juribašić Kulcsar, M.; Cindro, N.; Gracin, D.; 599 Biliškov, N.; Borovina, M.; Curić, M.; Halasz, I.; Užarević, K. Chem. 600 Commun. 2015, 51, 8058-8061.

601 (41) Belenguer, A. M.; Lampronti, G. I.; Cruz-Cabeza, A. J.; Hunter, 602 C. A.; Sanders, J. K. M. Solvation and surface effects on polymorph 603 stabilities at the nanoscale. Chem. Sci. 2016, 7, 6617-6627.

604 (42) Etter, M. C.; Reutzel, S. M.; Choo, C. G. Self-organization of 605 adenine and thymine in the solid state. J. Am. Chem. Soc. 1993, 115, 606 4411-4412.

607 (43) Caira, M. R.; Nassimbeni, L. R.; Wildervanck, A. F. Selective 608 formation of hydrogen bonded cocrystals between a sulfonamide and 609 aromatic carboxylic acids in the solid state. J. Chem. Soc., Perkin Trans. 6102 1995, 2, 2213-2216.

611 (44) Abourahma, H.; Urban, J. M.; Morozowich, N.; Chan, B. 612 Examining the robustness of a theophylline cocrystal during grinding 613 with additives. CrystEngComm 2012, 14, 6163-6169.

614 (45) Fischer, F.; Joester, M.; Rademann, K.; Emmerling, F. Survival 615 of the Fittest: Competitive Co-crystal Reactions in the Ball Mill. Chem. 616 - Eur. J. 2015, 21, 14969-14974.
(46) Berry, D. J.; Seaton, C. C.; Clegg, W.; Harrington, R. W.; Coles, 617 S. J.; Horton, P. N.; Hursthouse, M. B.; Storey, R.; Jones, W.; Friščić, 618 T.; Blagden, N. Applying Hot-Stage Microscopy to Co-Crystal 619 Screening: A Study of Nicotinamide with Seven Active Pharmaceutical 620 Ingredients. Cryst. Growth Des. 2008, 8, 1697-1712. 621

(47) Ojala, W. H.; Etter, M. C. Polymorphism in anthranilic acid: a 622 reexamination of the phase transitions. J. Am. Chem. Soc. 1992, 114, 623 10288-10293.

624

(48) Bučar, D.-K.; Day, G. M.; Halasz, I.; Zhang, G. G. Z.; Sander, J. 625 R. G.; Reid, D. G.; MacGillivray, L. R.; Duer, M. J.; Jones, W. The 626 curious case of (caffeine).(benzoic acid): how heteronuclear seeding 627 allowed the formation of an elusive cocrystal. Chem. Sci. 2013, 4, 628 4417-4425.

629

(49) Lončarić, I.; Popović, J.; Despoja, V.; Burazer, S.; Grgičević, I.; 630 Popović, D.; Skoko, Ž. Reversible Thermosalient Effect of N'-2- 631 Propylidene-4-hydroxybenzohydrazide Accompanied by an Immense 632 Negative Compressibility: Structural and Theoretical Arguments 633 Aiming toward the Elucidation of Jumping Phenomenon. Cryst. 634 Growth Des. 2017, 17, 4445-4453.

635

(50) Reilly, A. M.; et al. Report on the sixth blind test of organic 636 crystal structure prediction methods. Acta Crystallogr., Sect. B: Struct. 637 Sci., Cryst. Eng. Mater. 2016, 72, 439-459.

638

(51) Berland, K.; Hyldgaard, P. Exchange functional that tests the 639 robustness of the plasmon description of the van der Waals density 640 functional. Phys. Rev. B: Condens. Matter Mater. Phys. 2014, 89, 035412. 641

(52) Berland, K.; Arter, C. A.; Cooper, V. R.; Lee, K.; Lundqvist, B. I.; 642 Schroder, E.; Thonhauser, T.; Hyldgaard, P. van der Waals density 643 functionals built upon the electron-gas tradition: Facing the challenge 644 of competing interactions. J. Chem. Phys. 2014, 140, 18A539. 645

(53) Brown-Altvater, F.; Rangel, T.; Neaton, J. B. Ab initio phonon 646 dispersion in crystalline naphthalene using van der Waals density 647 functionals. Phys. Rev. B: Condens. Matter Mater. Phys. 2016, 93, 648 195206.

649

(54) Rangel, T.; Berland, K.; Sharifzadeh, S.; Brown-Altvater, F.; Lee, 650 K.; Hyldgaard, P.; Kronik, L.; Neaton, J. B. Structural and excited-state 651 properties of oligoacene crystals from first principles. Phys. Rev. B: 652 Condens. Matter Mater. Phys. 2016, 93, 115206.

(55) Taylor, R; Kennard, O. Hydrogen-bond geometry in organic 654 crystals. Acc. Chem. Res. 1984, 17, 320-326.

(56) Etter, M. C. Encoding and decoding hydrogen-bond patterns of 656 organic compounds. Acc. Chem. Res. 1990, 23, 120-126.

(57) Halasz, I. Single-Crystal-to-Single-Crystal Reactivity: Gray, 658 Rather than Black or White. Cryst. Growth Des. 2010, 10, 2817-2823. 659

(58) Coquerel, G. The structural purity of molecular solids-An 660 elusive concept? Chem. Eng. Process. 2006, 45, 857-862.

(59) Kieffer, J.; Karkoulis, D. PyFAI, a versatile library for azimuthal 662 regrouping. J. Phys.: Conf. Ser. 2013, 425, 202012.

(60) Hill, R. J.; Howard, C. J. Quantitative phase analysis from 664 neutron powder diffraction data using the Rietveld method. J. Appl. 665 Crystallogr. 1987, 20, 467-474.

(61) Caprio, M. LevelScheme: A level scheme drawing and scientific 667 figure preparation system for Mathematica. Comput. Phys. Commun. 668 2005, 171, 107-118.

669

(62) Sonneveld, E. J.; Visser, J. W. Automatic collection of powder 670 data from photographs. J. Appl. Crystallogr. 1975, 8, 1-7. 671

(63) MATLAB, version R2016a; The MathWorks Inc.: Natick, MA, 672 2016.

(64) Giannozzi, P.; et al. QUANTUM ESPRESSO: a modular and 674 open-source software project for quantum simulations of materials. J. 675 Phys.: Condens. Matter 2009, 21, 395502.

(65) Garrity, K. F.; Bennett, J. W.; Rabe, K. M.; Vanderbilt, D. 677 Pseudopotentials for high-throughput DFT calculations. Comput. 678 Mater. Sci. 2014, 81, 446-452. 